\title{
Study of the immunogenicity of recombinant protein consisting of multiple epitopes of porcine epidemic diarrhea virus highly expressed in Sf9 cells
}

\section{Yangkun Liu}

Nanyang Normal University

Xiaomin Hu

Nanyang Normal University

Nannan Zhang

Nanyang Normal University

$\mathrm{Na} \mathrm{Li}$

Nanyang Normal University

Zhihan Guan

Southwest University

Andrey Burov

Russian Academy of Science

Alexey Puzyr

Russian Academy of Science

Vladimir Bondar

Russian Academy of Science

Lunguang Yao ( $\sim$ lunguangyao@163.com )

Nanyang Normal University

\section{Research article}

Keywords: Porcine epidemic diarrhea virus, S1CD protein, baculovirus expression system, immunogenicity

Posted Date: July 27th, 2020

DOI: https://doi.org/10.21203/rs.3.rs-46171/v1

License: (1) (1) This work is licensed under a Creative Commons Attribution 4.0 International License.

Read Full License 


\section{Abstract \\ Background}

Current inactivated and attenuated vaccines can not provide complete protection, resulting in the ongoing epidemic of porcine epidemic diarrhea virus (PEDV), which has caused significant economic losses in the swine industry worldwide. To develop an alternative efficient and economical vaccine, a fusion protein named S1CD containing multiple neutralizing epitopes of PEDV was designed and expressed in Sf9 cells. The expression yield was optimized by production of baculoviral constructs harboring multiple S1CDexpressing cassettes, and the immunogenicity of the purified protein was evaluated.

\section{Results}

The results showed that high-level expression of the S1CD fusion protein was achieved by multi-copy gene co-expression. The recombinant virus carrying three copies of S1CD gene under the control of p10 promoter showed the highest expression level, with a yield of $462 \mathrm{mg} / \mathrm{L}$, which is about 2.6 or 3.5 fold that of the recombinant viruses carrying only one copy. BALB/c mice were subcutaneously immunized with purified S1CD protein three times at 2 week intervals. After immunization, S1CD protein could induce the mice produce high level of specific IgG antibodies and neutralizing antibodies.

\section{Conclusion}

Fusion S1CD protein consisting of multiple epitopes of porcine epidemic diarrhea virus was highly expressed by multi-copy gene co-expression. Moreover, it stimulated mice to produce high level of specific IgG antibodies and neutralizing antibodies. The S1CD protein expressed in this study could be used as a putative economic and efficient subunit vaccine against PEDV infection.

\section{Background}

Porcine epidemic diarrhea (PED) is a highly contagious intestinal disease, which is characterized by acute watery diarrhea and vomiting followed by severe dehydration leading to death, with a mortality rate of up to $90 \%$ in newborn piglets ${ }^{[1]}$. This disease was first reported in England in 1971 and subsequently spread to other European countries and most of the Asian swine raising countries ${ }^{[2]}$. Since October 2010, outbreaks of highly virulent PED caused by new variant strains have been reported almost all over the world $^{[3-5]}$. Currently, although pigs have been immunized with commercial PED vaccines, which mostly include inactivated and attenuated vaccines, PED still frequently broke out and caused significant economic losses to the global swine industry ${ }^{[6,7]}$. Therefore, it is important to develop an effective vaccine for the prevention of PED. 
Porcine epidemic diarrhea virus (PEDV), the causative agent of PED, belongs to the genera Alphacoronavirus, the family Coronaviridae, and the order Nidovirales ${ }^{[8]}$. It is a pleomorphic enveloped, single-stranded positive-sense RNA virus, with a genome size of about $28 \mathrm{~kb}^{[9]}$. The virus possesses four structural proteins including spike protein $(S)$, membrane protein $(M)$, envelope protein $(E)$, and nucleocapsid protein $(\mathrm{N})^{[10]}$. Among the structure proteins, the $S$ protein contains both the receptor binding domain, which mediates virus-host recognition and virus internalization, and the antigenic epitope, which is responsible for inducing neutralizing antibody (NA ${ }^{[11]}$. Therefore, the $S$ protein is the principle antigenic determinant and has the potential to be an ideal vaccine candidate ${ }^{[12]}$.

The S protein can be divided into two domains: S1 (1 789aa) and S2 (790 1383aa) ${ }^{[13]}$. Previous work has shown that the $S 1$ domain contains the main neutralizing epitopes, including the CO-26K-equivalent epitope (COE), and the S1D domain ${ }^{[14]}$. The COE epitope (499 638aa), which corresponds to the collagenase- digested fragment (namely CO-26K) of transmissible gastroenteritis virus (TGEV), has been considered an ideal antigenic candidate for development of subunit vaccine ${ }^{[15,16]}$. In addition, previous studies have shown that the epitope region designated S1D (636 789aa) in the S1 domain has the capacity to induce neutralization antibodies against PEDV. Moreover, the immune serum against the two linear epitopes of S1D domain, namely S1D5 (744 759aa) and S1D6 (756 771 aa), could react with the native $S$ protein of PEDV ${ }^{[17]}$. Therefore, due to above-mentioned studies in the neutralizing antigenic epitopes of S1 domain, a multiepitope-based fusion protein (S1CD) containing COE, S1D5 and S1D6 epitope may be appropriate targets for developing subunit vaccine against PEDV.

Baculovirus expression vector system (BEVS) has been widely utilized as a vaccine expression/delivery vector $^{[18,19]}$. It shows many advantages over other expression systems, such as large cloning capacity, proper post-translation modifications, high titers and biosafety, and strong induction innate immune respons $\mathrm{e}^{[20]}$. Therefore, the baculovirus expression system is a promising platform for the development of subunit vaccines in humans and animals. However, a main limitation of BEVS is the comparatively low protein yield compared with E.coli or yeast expression system. Here we report that application of multicopy gene co-expression in BEVS permits substantially improved S1CD protein production in Sf9 cells. We also evaluated the immunogenicity of purified S1CD protein in BALB/c mice.

\section{Results}

\section{Generation and Characterization of recombinant baculoviruses}

The recombinant bacteria carrying positive bacmids was used to infect Sf9 cells to produce recombinant baculovirus as described previously ${ }^{[21]}$. The expression of mCherry gene was used to trace proper viral infection. When examined by fluorescence microscopy, it was found that obvious cytopathic effect (CPE) and red fluorescence in virus-infected Sf9 cells. The presence of the S1CD gene in recombinant viruses was confirmed by direct PCR of the viral genomic DNA. As expected, PCR amplification of the recombinant viral genomic DNA revealed a specific band of 600 bp. Non-specific amplification was not 
observed in the negative control sample (Fig 1). These results indicated that S1CD gene was inserted into recombinant baculovirus.

\section{Expression and identification of recombinant S1CD protein}

Sf9 cells were infected with eight recombinant baculoviruses, respectively, at a $\mathrm{MOI}$ of 10 for protein expression. At 72 h.p.i, the expression of S1CD protein in different virus-infected insect cells was first determined by IFA. Since virus-infected Sf 9 cells exhibited red fluorescence, immunofluorescence in the infected Sf9 cells was monitored by incubation with FITC-labeled antibody. As shown in Fig 2A, the green fluorescence indicated that eight recombinant baculoviruses expressed S1CD protein in Sf9 cells. Further analysis by western blot showed that a specific band appeared at $23 \mathrm{kDa}$, which correlated with the expected size of S1CD protein (Fig 2B). Thus, the experimental results suggest that the S1CD protein was expressed in the Sf9 cells. In addition, the S1CD protein was also detected clearly in the cell culture supernatant by the SDS-PAGE and western blot (Fig 2C and Fig 2D), which indicated that the S1CD protein could be released into the cell culture supernatant.

\section{Gene expression yield analysis}

Eight recombinant baculoviruses harboring from one to four repeats of S1CD-expressing cassettes under the control of the polh promoter, or the p10 promoter, or both promoters were constructed and used to infect Sf9 cells at a MOI of 5. At the transcriptional level, qPCR showed that the expression levels of S1CD mRNA increased with the increasing number of expression cassettes in baculovirus (Fig 3A). However, the protein expression level of Ac-IM-p10-S1CD, Ac-IM-2p10-2S1CD, Ac-IM-3p10-3S1CD, Ac-IM-ph-S1CD, AcIM-2ph-2S1CD, Ac-IM-3ph-3S1CD, Ac-IM-php10-2S1CD, Ac-IM-2ph2p10-4S1CD were $176 \mathrm{mg} / \mathrm{L}, 318 \mathrm{mg} / \mathrm{L}$, $462 \mathrm{mg} / \mathrm{L}, 131 \mathrm{mg} / \mathrm{L}, 245 \mathrm{mg} / \mathrm{L}, 345 \mathrm{mg} / \mathrm{L}, 294 \mathrm{mg} / \mathrm{L}, 327 \mathrm{mg} / \mathrm{L}$, respectively (Fig 3B). The maximum yield of S1CD protein in Sf9 cells were obtained from the recombinant baculovirus harboring three-copy tandem S1CD-expressing cassettes driven by the p10 promoter, Ac-IM-3p10-3S1CD. The yield reached $462 \mathrm{mg} / \mathrm{L}$, which is 2.6-fold higher than that of Ac-IM-p10-S1CD, and 3.5-fold higher than than that AcIM-ph-S1CD. Therefore, Ac-IM-3p10-3S1CD was used as seed virus to infect Sf9 cells in the following experiments.

\section{Purification of recombinant protein S1CD}

$4.0 \times 10^{8}$ of Sf9 cells were infected with Ac-IM-3p10-3S1CD at a MOI of 5 for $96 \mathrm{~h}$. The purification of recombinant S1CD protein from the cell lysate was facilitated by the presence of a $6 \times$ His-tag at the Cterminal. The recombinant protein was purified by $\mathrm{Ni}^{2+}$-affinity chromatography, separated on $12 \% \mathrm{SDS}$ PAGE and stained with Coomassie blue to evaluate the purity of the expressed S1CD protein. As shown in Fig 4, a specific band at $23 \mathrm{kDa}$ was observed in the elution buffer, and the protein obtained was highly pure based on Coomassie blue staining.

\section{Antibody responses against PEDV in immunized mice}


Specific IgG antibodies were detected in serum samples collected at different days post primary immunization using an indirect ELISA. As shown in Fig 5A, the S1CD protein group and the commercial PEDV inactivated vaccine group exhibited detectable specific IgG antibody titers initially at $14 \mathrm{dpi}$ and increased gradually over time. The specific IgG antibody titers of the S1CD protein group and the commercial vaccine group were significantly higher than those of the PBS group at $14 \mathrm{dpi}(P<0.05), 28$ dpi $(P<0.01)$ and $42 \mathrm{dpi}(P<0.01)$. Meanwhile, the specific IgG antibody titer of the S1CD protein group was slightly lower than that of the commercial inactivated vaccine group at 14,28 , and $42 \mathrm{dpi}$, but there was no significant difference between them $(P>0.05)$.

The ability of the purified S1CD protein to induce PEDV-neutralizing antibodies was determined using a virus neutralization assay. As shown in Fig 5B, immunization with S1CD protein and commercial PEDV inactivated vaccine elicited neutralizing antibodies against PEDV in all immunization mice, and no significant difference was observed between them $(P>0.05)$. Meanwhile, no neutralizing antibody titers were detected in the mice of the PBS groups (Fig 7B). Together, these results indicated that the purified S1CD protein could induce a strong humoral immune response in mice.

\section{Discussion}

In recent years, the ongoing epidemic of PEDV has caused significant economic losses to the swine industry in Asia as well as Europe and North America ${ }^{[22]}$. To date, there have been several PEDV commercial vaccines in the market, mainly including inactivated vaccines and attenuated vaccines. However, for some reason unknown, none of these vaccines has provided complete protection in piglets $^{[23]}$. Hence, it is of vital importance and a hot research topic to develop a new, efficient and economical vaccine to protect against PEDV infection.

Currently, several PEDV subunit vaccines, which mainly focus on the S1 domain, have been constructed and shown to induce protective immunity in piglets ${ }^{[24-26]}$. As the main neutralization epitope in the S1 domain, COE and S1D have the capacity to induce effective neutralization antibodies against PEDV, thus may be the ideal antigenic candidate for the development of subunit vaccine ${ }^{[15,27-29]}$. Moreover, previous studies implied that the immunogenicity of a single antigen epitope is weak and the protective effect is incomplete, and the combination of multiple antigen epitopes can enhance the immunogenicity ${ }^{[30]}$. For this reason, we evaluated synergistic effects between the COE and S1D through fusion of the proteins. Therefore, in this study, a fusion gene encoding S1CD protein containing multiple neutralizing epitope of S1 domain (COE, S1D5 and S1D6) was synthesized and high-yielding expressed, and its immunogenicity as a subunit vaccine candidate was evaluated in BALB/c mice.

Previous studies reported that full length or truncated S1 protein of PEDV could be produced in various expression systems, including E. coli, Lactobacillus, yeast, mammalian cell and baculovirus ${ }^{[26,31-34]}$. Among these expression systems, baculovirus expression system is a powerful platform for commercial production of subunit vaccines (Cervarix ${ }^{\circledR}$, GlaxoSmithKline; Ingelvac CircoFLEX ${ }^{\circledR}$, Boehringer Ingelheim) and has also been used for the production of antigen proteins of various viruses ${ }^{[35]}$. In addition, the S1 
protein has abundant potential glycosylation sites which is conducive to maintain its biological function ${ }^{[36]}$. Thus, BEVS was chosen to express the S1CD protein that may require complex folding machinery and post-translational modifications to maintain its naturally occurring conformation. IFA and western blot results showed that S1CD protein was expressed in the Sf9 cells. Surprising, the S1CD protein was also detected in the cell culture supernatant, indicating that the S1CD protein could be secreted into the medium. However, the prediction result of SignaIP online predictive sites shows that there is no signal peptide sequence in the S1CD protein. The detailed mechanism of S1CD protein secretion into the medium is still unclear, which needs to be further explored.

The protein yield using conventional BEVS is usually relatively low, which represents a bottleneck to commercial applications ${ }^{[37]}$. To improve the yield of S1CD protein, we designed a panel of baculoviral constructs which containing from one to four repeats of S1CD-expressing cassettes under the control of the polh promoter, or the 10 promoter, or both promoters. The results suggested that the number of expression cassettes has a positive effect on the mRNA level, however, an increase in the number of expression cassettes did not always enhance its protein expression level, which indicated that increasing the number of expression cassettes can improve the transcription of mRNA but not always enhanced the translation level. It is still unclear as to why such phenomenon occurred here, however, some studies have reported similar conclusion in Pichia pastoris ${ }^{[38]}$. We speculated that possibly high copy number may exert severe stress on the endoplasmic reticulum (ER) and disturb the metabolic balance, thereby affecting exogenous protein expression ${ }^{[39]}$. In addition to the number of expression cassette, promoter also affect the protein yield, as the expression level of S1CD protein driven by the p10 promoter was higher than that of polh promoter, when the number of expression cassettes was equal. These results indicated that appropriate promoter and expression cassette number could contribute to increase the protein production in Sf9 cells. In this study, the highest yield obtained was $462 \mathrm{mg} / \mathrm{L}$, which is an acceptable amount of recombinant protein in terms of the possibility of producing a novel vaccine.

The PEDV-specific IgG antibody and neutralizing antibody (NA) in the serum of mice immunized with purified S1CD fusion protein were evaluated compare to that in mice of inactivated vaccine-immunized group and PBS-immunized group, which indicates humoral immune response. The serum isolated from mice immunized with purified S1CD protein showed significant higher PEDV-specific IgG antibodies and neutralizing antibodies compared to that in the PBS-immunized mice $(P<0.05)$, and slightly lower than that in inactivated vaccine-immunized mice $(P>0.05)$, indicating that $S 1 C D$ protein induced a high level of immune response. These results were consistent with the previous studies, which reported that epitope 499-600 (COE), 744-759 (S1D5) and 756-771 (S1D6) might represent the immunodominant neutralizing epitope of PEDV ${ }^{[40]}$. Considering that subunit vaccine can solve some problems experienced with inactivated vaccine, which cannot distinguish between infected and vaccinated animals and has biosafety concerns, the purified S1CD protein have been suggested as an alternative to develop a subunit vaccine against PEDV. However, as the BALB/c mice is not a susceptible animal model for PEDV, we cannot use it to carry out challenge experiments to evaluate the protection efficacy of S1CD protein. On 
the basis of this study, our further studies will include usage of pigs to investigate the host immunity and protection induced by the S1CD protein to develop effective subunit vaccines for the prevention of PED.

\section{Conclusions}

In this study, the S1CD fusion gene containing multiple neutralizing epitopes of PEDV (COE, S1D5 and S1D6) was synthetized, and its expression yield in baculovirus expression system was optimized by multi-copy gene expression. The mice immunized with purified S1CD protein elicited high level of specific IgG antibodies and neutralizing antibodies, suggesting the possibility of a subunit vaccine against PEDV infection.

\section{Methods}

\section{Virus, Cell line, bacterial strains and plasmid}

PEDV HeN170821 (GenBank accession No: MK862249.1) was isolated from the intestines of piglets with severe diarrhea in Henan, China, and was maintained in our laboratory. Vero cells were cultured in DMEM (Gibco, USA) in a culture bottle at $37^{\circ} \mathrm{C}$ and $5 \% \mathrm{CO}_{2}$. Sf9 cells were grown and maintained at $27^{\circ} \mathrm{C}$ in $\mathrm{Sf}-$ $900^{\mathrm{TM}} \otimes \mathrm{SFM}$ medium (Thermo Fisher Scientific, USA). Transfer vector PYBDM-IM (a mcherry fragment driven by IRES is insert into the $S p h$ and $K p n l$ sites of pFBDM), and $E$. coli strains AcMultiBacmid/rSW106/asd $/ /$ inv $^{+}$had been constructed previously ${ }^{[21]}$.

\section{Construction of transfer vectors}

The nucleotide sequence of S protein of PEDV CV777 strain (GenBank accession No. JN599150.1) was used as a reference to synthesize COE (nt 1495-1914) gene, and D56 (nt 2230-2313) gene. In this study, we fused D56 gene along with a $6 \times$ His-tag to the $C$ terminus of COE gene with the flexible linker (GGGGS) ${ }_{3}$ to generate a novel fusion gene, denoted as S1CD (Fig 6). The S1CD gene was synthesized, and optimized for expression in Sf9 cells by Sangon Biotech (Shanghai, China). The codon-optimized S1CD gene was cloned into plasmid pYBDM-IM, thereby producing a series of recombinant transfer vectors, which comprising S1CD gene under the control of different promoter or promoter combinations (Fig 7). All of the constructed plasmids were verified by nucleotide sequencing.

\section{Generation of recombinant baculoviruses}

The recombinant transfer vectors were transformed into $E$. coli AcMultiBacmid/ rSW106/asd $/ \mathrm{inv}^{+}$ competent cells through mini-Tn7 transposition to generate recombinant bacmids. Then, $E$. coli AcMultiBacmid/rSW106/asd $/$ inv $^{+}$cells with recombinant S1CD gene were cultured in LB broth until

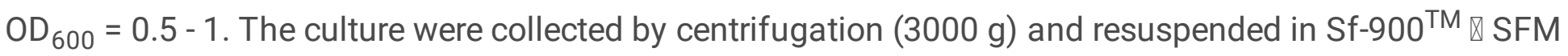
medium. The bacterial suspension was adjusted to different densities $\left(10^{5}-10^{8}\right.$ cells $\left./ \mathrm{mL}\right)$. Sf9 cells at $10^{5} / \mathrm{mL}$ were incubated overnight in 24 -well plates ( $70-80 \%$ confluent single layer). After removing the 
medium, $500 \mu \mathrm{L}$ bacterial cells at different concentrations were added to each well to infect Sf9 cells $^{[21]}$. After culturing at $27^{\circ} \mathrm{C}$ for $4-5 \mathrm{~h}$, each well was washed three times. Finally, $500 \mu \mathrm{L}$ fresh Sf- $900^{\mathrm{TM}} \otimes \mathrm{SFM}$ medium was added and incubated for 3-5 days. The transfected cells were lysed by 3 freeze-thaw cycles and the supernatant containing viruses was collected for further infection. After 2 passages in Sf9 cells, the generated viruses were harvested and detected with PCR using S1CD gene-specific primers (S1CD F: 5'-CTCGAGATGA CTTTGCCATCATTCA -3'; S1CD R: 5'-GCTAGCTTAGTGGTGGTGGTGATGGT -3'). The titers of the various viruses constructed were measured by plaque analysis.

\section{Immunofluorescence identification}

An indirect immunofluorescence assay (IFA) was performed to determine whether the desired proteins were expressed in the Sf9 cells infected with different recombinant baculoviruses. Briefly, Sf9 cells were seeded in 24-well cell culture plates $\left(2.0 \times 10^{5}\right.$ cells/well $)$ and infected with recombinant baculoviruses. Cells were fixed with $4 \%(\mathrm{v} / \mathrm{v})$ paraformaldehyde at $48 \mathrm{~h}$ post infection (h.p.i) and washed three times with PBS containing $0.05 \%$ Tween (PBST). Immunostaining was performed with mouse anti-His antibody (Boshide, Wuhan, China) as primary antibody and FITC-labeled goat anti mouse IgG antibody (Invitrogen, China) as secondary antibody. Cells were washed five times with PBST after incubation with each antibody. Digital images were captured using fluorescence microscopy.

\section{ELISA and Western blot analysis}

To further evaluate S1CD protein expression level, Sf9 cells were cultured in $50 \mathrm{~mL} \mathrm{Sf-} 900^{\mathrm{TM}} \otimes \mathrm{SFM}$ medium at $27^{\circ} \mathrm{C}$ with shaking $(125 \mathrm{rpm})$ and then inoculated with different recombinant baculoviruses at a multiplicity of infection (MOI) of 5, respectively. After 96 h.p.i, the culture was separated into cells and supernatant at $12000 \mathrm{~g}$ for $10 \mathrm{~min}$. The total protein concentration of the S1CD protein in the cells and supernatant was determined by the His Tag ELISA Detection Kit (GenScript, Nanjing, China). For western blot analysis, the cells and supernatant samples were separated on $12 \%$ SDS-PAGE gels, respectively, then transferred to polyvinylidene fluoride (PVDF) membranes (Merck Millipore, USA) and blocked with $5 \%(\mathrm{w} / \mathrm{v})$ nonfat milk in PBST for $1 \mathrm{~h}$ at $37^{\circ} \mathrm{C}$. The membranes were incubated overnight with mouse antiHis monoclonal antibody at $4^{\circ} \mathrm{C}$, rinsed with PBST, and incubated with HRP-conjugated goat anti mouse IgG antibody (Boshide, Wuhan, China). After five washes with PBST for 5 min each time, detection was performed with DAB (3, 3'-diaminobenzidine) solution (Boshide, Wuhan, China).

\section{Quantitative real-time PCR analysis}

To test the differences in S1CD transcripts between Sf9 cells infected with different viruses, quantitative real-time PCR (qPCR) analysis was performed. Total RNA was extracted from virus infected-Sf9 cells and quantified by spectrophotometry. $500 \mathrm{ng}$ of total RNA from each sample was used as a template to synthesize cDNA according to the PrimeScript ${ }^{T M}$ II 1st Strand cDNA Synthesis Kit's instructions (TaKaRa). The synthesized cDNA was used for qPCR analysis of S1CD transcript levels. In qPCR, the primer pairs used for a 145 bp amplicon of S1CD gene were S1F: 5'-GTCATAGTGGTGCCAACCT-3' and S1R: 5'- 
TTACTATCCTGTGACTTAGA-3'. An internal system control with the primer pair of 28S F: 5'CTGGCTTGATCCAGA

TGTTCAG-3'and 28S R: 5'-GGATCGATAGGCCGTGCTT-3' for the Sf9 cell 28S rRNA was used to normalize the reactions. The relative transcript level of each gene was calculated according to the $2^{-\mathrm{Ct}}$, for unnormalized genes, and the $2^{-\Delta \Delta C t}$ method, for the genes normalized to $28 \mathrm{~S}$. The relative transcript levels in the Ac-IM-p10-S1CD infection sample was considered to be 1 and the relative transcript levels of other samples was calculated accordingly. Each of the amplifications was run in triplicates to calculate experimental variance for statistical analysis by the Student's T-test.

\section{Purification of S1CD protein}

$4.0 \times 10^{8}$ of Sf9 cells were grown in $100 \mathrm{~mL}$ volumes in $500 \mathrm{~mL}$ polycarbonate Erlenmeyer flasks and incubated in an orbital shaker incubator at $125 \mathrm{rpm}$ and $27^{\circ} \mathrm{C}$. The initial cell density was $2.0 \times 10^{5}$ cells $/ \mathrm{mL}$. The Sf9 cells were infected with the recombinant baculoviruses, Ac-IM-3p10-3S1CD, at a MOI of 5. At 96 h.p.i, the cells were harvested by centrifugation $\left(1000 \mathrm{~g}, 10 \mathrm{mins}, 4^{\circ} \mathrm{C}\right)$ and lysed by sonication. The lysed Sf 9 cells were centrifuged at $12,000 \mathrm{~g}$ for $10 \mathrm{~min}$, the supernatant was purified using Ni-NTA agarose according to the manufacturer's instructions.

\section{Immunization of mice}

Six-week-old female specific pathogen-free (SPF) BALB/c mice were purchased from Autobio Diagnostics Co., Ltd in Henan, China, and kept under SPF conditions with free access to standard water and diet. All animal procedures were approved by the Ethical Committee for Animal Experiments (Nanyang Normal University, China). The mice were randomly divided into three groups (G1-G3) each with 10 mice. Group 1 were immunized subcutaneously with $100 \mu \mathrm{g}$ of purified S1CD protein in the presence of Freund's complete adjuvant (Sigma, USA) and boosted twice in the presence of Freund's incomplete adjuvant at 2week intervals. Group 2 were immunized subcutaneously with equivalent volumes of adjuvant as a negative control. Group 3 were immunized subcutaneously with equivalent volumes of the commercial PEDV inactivated vaccine (inactivated virus of PEDV CV777 strain; virus titer before inactivation $\geq 10^{7}$ $\left.T C I D_{50}\right)$. Serum samples from all groups were collected at $0,14,28$, and 42 days post primary immunization (dpi) for the antibody detection and serum neutralization assay. At the end of experiment, animals were euthanized by the intravenous administration of an overdose of sodium pentobarbital.

\section{Analysis of antibody levels}

An indirect ELISA was performed to determine the levels of IgG antibodies in the serum. Briefly, $100 \mu \mathrm{L}$ of purified S1 protein ( $1 \mu \mathrm{g} / \mathrm{mL}$, Genscript, China), which expressed as a recombinant protein in $E$. coli, was added to each well of the 96 -well plate and incubated overnight at $4{ }^{\circ} \mathrm{C}$. After blocking with PBS containing $1 \%$ bovine serum albumin, serum samples were serially diluted, added to the corresponding wells in triplicate and incubated at $37^{\circ} \mathrm{C}$ for $1 \mathrm{~h}$. Then, HRP conjugated goat anti-mouse IgG antibodies were added to each well and incubated for $1 \mathrm{~h}$ at $37^{\circ} \mathrm{C}$. Plates were incubated with TMB substrate 
solution (Sigma, USA) for 15 min in the dark. The reactions were terminated with $2 \mathrm{M} \mathrm{H}_{2} \mathrm{SO}_{4}$ and the wells read at $450 \mathrm{~nm}$. Serum samples from Balb/C mouse received PBS as blank control (background). Endpoint titers were defined as the highest dilution that gave an absorbance two times higher than the background.

\section{Neutralization assay}

To determine whether mice generated PEDV-neutralizing antibody, sera (1: 100 dilution, $50 \mu \mathrm{L})$ from mice of each group were mixed with an equal volume of PEDV HeN170821 $\left(10^{5} \mathrm{pfu} / \mathrm{mL}\right)$ at $37^{\circ} \mathrm{C}$. After $1 \mathrm{~h}$ incubation, the treated viruses were used to infect Vero cells in 96-well plates. The plates were incubated at $37^{\circ} \mathrm{C}$ in a $5 \% \mathrm{CO}_{2}$ atmosphere and examined daily for 3 days for PEDV-specific cytopathic effects (CPE). The Reed - Muench statistical method was used to calculate the results.

\section{Statistical analysis}

Data were expressed as the means \pm SEM of three replicates per test in a single experiment. The differences between each group were compared by one-way analysis of variance (ANOVA), combined with Tukey's post hoc test. $P<0.05$ was considered statistically significant and $P<0.01$ was considered highly significant.

\section{Abbreviations}

BEVS: Baculovirus Expression Vector System; DAB:3,3' -diaminobenzidine; DAP:2,6-diaminopimelic; dpi:days post-immunization; ELISA:Enzyme linked immunosorbent assay; hpi:hours post infection; IFA:indirect immunofluorescence assay; MOI:multiplicity of infection; PEDV:porcine epidemic diarrhea virus; NA:neutralizing antibody; SDS-PAGE:sodium dodecyl sulfate-polyacrylamide gel electrophoresis; SPF:specific pathogen-free

\section{Declarations}

\section{Ethics approval and consent to participate}

This procedure was approved by the Nanyang Normal University (Belongs to Chinese Association for Laboratory Animal Sciences, CALAS). All animals were handled strictly in accordance with the good animal practice in order to minimize animal sufferings during blood sampling. Finally, the mice were euthanized by the intravenous administration of an overdose of sodium pentobarbital.

\section{Consent for publication}

Not applicable.

\section{Availability of data and materials}


The datasets used and/or analysed during the current study are available from the corresponding author on reasonable request.

\section{Competing interests}

The authors declare that they have no competing interests. The work is an original paper and is not under consideration in other journals.

\section{Funding}

This work was financially supported by the National Natural Science Foundation of China (No. 31870917), The program for Innovative Research Team of Science and Technology in University of Henan Province (No. 20IRTSTHN024), the fund of Scientific and Technological research project of Henan province (No. 202102110097 and 182102310077), Key Scientific Research Projects of Colleges and Universities in Henan Province of China (No. 19A230002 and 18A230011) and the Foundation of Nanyang Normal University (No. 2017ZX012)The funding bodies played no role in the design of the study, the collection, analysis, and interpretation of data and in writing the manuscript.

\section{Authors' contributions}

YLG directed the project and participated in the coordination and management of the study. YLG and LYK designed the study. HXM analyzed the data. LYK and ZNN performed the experiments. BA, PA and BV guided the protein purification and drafted the manuscript. All authors read and approved the final manuscript.

\section{Acknowledgments}

Not Applicable.

\section{References}

1. Hsueh FC, Lin CN, Chiou HY, Chia MY, Chiou MT, Haga T, Kao CF, Chang YC, Chang CY, Jeng CR, Chang HW. Updated Phylogenetic Analysis of the Spike Gene and Identification of a Novel Recombinant Porcine Epidemic Diarrhoea Virus Strain in Taiwan. Transbound Emerg Dis. 2020;67(1):417-30.

2. Poonsuk K, Zhang J, Chen Q, Gonzalez W, da Silva Carrion LC, Sun Y, Ji J, Wang C, Main R, Zimmerman J, Giménez-Lirola L. Quantifying the Effect of Lactogenic Antibody on Porcine Epidemic Diarrhea Virus Infection in Neonatal Piglets. Vet Microbiol. 2016;197:83-92.

3. Huang YW, Dickerman AW, Piñeyro P, Li L, Fang L, Kiehne R, Opriessnig T, Meng XJ. Origin, Evolution, and Genotyping of Emergent Porcine Epidemic Diarrhea Virus Strains in the United States. mBio. 2013;4(5):e00737-13. 
4. Sun J, Li Q, Shao C, Ma Y, He H, Jiang S, Zhou Y, Wu Y, Ba S, Shi L, Fang W, Wang X, Song H. Isolation and Characterization of Chinese Porcine Epidemic Diarrhea Virus With Novel Mutations and Deletions in the S Gene. Vet Microbiol. 2018;221:81-9.

5. Jung K, Saif LJ, Wang Q. Porcine Epidemic Diarrhea Virus (PEDV): An Update on Etiology, Transmission, Pathogenesis, and Prevention and Control. Virus Res. 2020;286:198045.

6. Diep NV, Sueyoshi M, Izzati U, Fuke N, Teh APP, Lan NT, Yamaguchi R. Appearance of US-like Porcine Epidemic Diarrhoea Virus (PEDV) Strains Before US Outbreaks and Genetic Heterogeneity of PEDVs Collected in Northern Vietnam During 2012-2015. Transbound Emerg Dis. 2018;65(1):e83-93.

7. Lin CM, Saif LJ, Marthaler D, Wang Q. Evolution. Antigenicity and Pathogenicity of Global Porcine Epidemic Diarrhea Virus Strains. Virus Res. 2016;226:20-39.

8. Jung K, Saif LJ. Porcine Epidemic Diarrhea Virus Infection: Etiology, Epidemiology, Pathogenesis and Immunoprophylaxis. Vet J. 2015;204(2):134-43.

9. Rasmussen TB, Boniotti MB, Papetti A, Grasland B, Frossard JP, Dastjerdi A, Hulst M, Hanke D, Pohlmann A, Blome S, van der Poel WHM, Steinbach F, Blanchard Y, Lavazza A, Bøtner A, Belsham GJ. Full-length Genome Sequences of Porcine Epidemic Diarrhoea Virus Strain CV777; Use of NGS to Analyse Genomic and Sub-Genomic RNAs. PLoS One. 2018;13(3):e0193682.

10. Kocherhans R, Bridgen A, Ackermann M, Tobler K. Completion of the Porcine Epidemic Diarrhoea Coronavirus (PEDV) Genome Sequence. Virus Genes. 2001;23(2):137-44.

11. Zhang Y, Zhang X, Liao X, Huang X, Cao S, Wen X, Wen Y, Wu R, Liu W. Construction of a Bivalent DNA Vaccine Co-Expressing $S$ Genes of Transmissible Gastroenteritis Virus and Porcine Epidemic Diarrhea Virus Delivered by Attenuated Salmonella Typhimurium. Virus Genes. 2016;52(3):354-64.

12. Huang KY, Yang GL, Jin YB, Liu J, Chen HL, Wang PB, Jiang YL, Shi CW, Huang HB, Wang JZ, Wang G, Kang YH, Yang WT, Wang CF. Construction and Immunogenicity Analysis of Lactobacillus Plantarum Expressing a Porcine Epidemic Diarrhea Virus S Gene Fused to a DC-targeting Peptide. Virus Res. 2018;247:84-93.

13. Li W, Wicht O, van Kuppeveld FJ, He Q, Rottier PJ, Bosch BJ. A Single Point Mutation Creating a Furin Cleavage Site in the Spike Protein Renders Porcine Epidemic Diarrhea Coronavirus Trypsin Independent for Cell Entry and Fusion. J Virol. 2015;89(15):8077-81.

14. Li C, Li W, Lucio de Esesarte E, Guo H, van den Elzen P, Aarts E, van den Born E, Rottier PJM, Bosch BJ. Cell Attachment Domains of the Porcine Epidemic Diarrhea Virus Spike Protein Are Key Targets of Neutralizing Antibodies. J Virol. 2017;91(12):e00273-17.

15. Wang X, Wang L, Huang X, Ma S, Yu M, Shi W, Qiao X, Tang L, Xu Y, Li Y. Oral Delivery of Probiotics Expressing Dendritic Cell-Targeting Peptide Fused With Porcine Epidemic Diarrhea Virus COE Antigen: A Promising Vaccine Strategy Against PEDV. Viruses. 2017;9(11):312.

16. Li Q, Peng O, Wu T, Xu Z, Huang L, Zhang Y, Xue C, Wen Z, Zhou Q, Cao Y. PED Subunit Vaccine Based on COE Domain Replacement of Flagellin Domain D3 Improved Specific Humoral and Mucosal Immunity in Mice. Vaccine. 2018;36(11):1381-8. 
17. Sun D, Feng L, Shi H, Chen J, Cui X, Chen H, Liu S, Tong Y, Wang Y, Tong G. Identification of Two Novel B Cell Epitopes on Porcine Epidemic Diarrhea Virus Spike Protein. Vet Microbiol. 2008;131(12):73-81.

18. Possee RD, Chambers AC, Graves LP, Aksular M, King LA. Recent Developments in the Use of Baculovirus Expression Vectors. Curr Issues Mol Biol. 2020;34:215-30.

19. Lin SY, Chung YC, Hu YC. Update on Baculovirus as an Expression and/or Delivery Vehicle for Vaccine Antigens. Expert Rev Vaccines. 2014;13(12):1501-21.

20. Premanand B, Zhong Wee P, Prabakaran M. Baculovirus Surface Display of Immunogenic Proteins for Vaccine Development. Viruses. 2018;10(6):298.

21. Yao LG, Sun JC, Xu H, Kan YC, Zhang XM, Yan HC. A novel economic method for high throughput production of recombinant baculovirus by infecting insect cells with Bacmid-containing diminopimelate-auxotrophic Escherichia coli. J Biotechnol. 2010;145(1):23-9.

22. Park JE, Jang H, Kim JH, Hyun BH, Shin HJ. Immunization With Porcine Epidemic Diarrhea Virus Harbouring Fc Domain of IgG Enhances Antibody Production in Pigs. Vet Q. 2020;40(1):183-9.

23. Li Y, Wang G, Wang J, Man K, Yang Q. Cell Attenuated Porcine Epidemic Diarrhea Virus Strain Zhejiang08 Provides Effective Immune Protection Attributed to Dendritic Cell Stimulation. Vaccine. 2017;35(50):7033-41.

24. Suzuki T, Terada Y, Enjuanes L, Ohashi S, Kamitani W. S1 Subunit of Spike Protein From a Current Highly Virulent Porcine Epidemic Diarrhea Virus Is an Important Determinant of Virulence in Piglets. Viruses. 2018;10(9):467.

25. Subramaniam S, Yugo DM, Heffron CL, Rogers AJ, Sooryanarain H, LeRoith T, Overend C, Cao D, Meng XJ. Vaccination of Sows With a Dendritic Cell-Targeted Porcine Epidemic Diarrhea Virus S1 Protein-Based Candidate Vaccine Reduced Viral Shedding but Exacerbated Gross Pathological Lesions in Suckling Neonatal Piglets. J Gen Virol. 2018;99(2):230-9.

26. Makadiya N, Brownlie R, van den Hurk J, Berube N, Allan B, Gerdts V, Zakhartchouk A. S1 Domain of the Porcine Epidemic Diarrhea Virus Spike Protein as a Vaccine Antigen. Virol J. 2016;13:57.

27. Tien NQ, Huy NX, Kim MY. Improved Expression of Porcine Epidemic Diarrhea Antigen by Fusion With Cholera Toxin B Subunit and Chloroplast Transformation in Nicotiana tabacum. Plant Cell Tissue Organ Cult. 2019;137(2):213-23.

28. Piao DC, Shin DW, Kim IS, Li HS, Oh SH, Singh B, Maharjan S, Lee YS, Bok JD, Cho CS, Hong ZS, Kang SK, Choi YJ. Trigger Factor Assisted Soluble Expression of Recombinant Spike Protein of Porcine Epidemic Diarrhea Virus in Escherichia Coli. BMC Biotechnol. 2016;16(1):39.

29. Li Q, Xu Z, Wu T, Peng O, Huang L, Zhang Y, Xue C, Wen Z, Zhou Q, Cao Y. A Flagellin-Adjuvanted PED Subunit Vaccine Improved Protective Efficiency Against PEDV Variant Challenge in Pigs. Vaccine. 2018;36(29):4228-35.

30. Zhang ZW, Zhang YG. Advances in Epitopes Vaccines against foot-and-mooth disease. Chinese Journal of Zoonoses. 2008;24(6):571-2. 
31. Piao DC, Lee YS, Bok JD, Cho CS, Hong ZS, Kang SK, Choi YJ. Production of Soluble Truncated Spike Protein of Porcine Epidemic Diarrhea Virus From Inclusion Bodies of Escherichia Coli Through Refolding. Protein Expr Purif. 2016;126:77-83.

32. Liu DQ, Ge JW, Qiao XY, Jiang YP, Liu SM, Li YJ. High-level Mucosal and Systemic Immune Responses Induced by Oral Administration With Lactobacillus-expressed Porcine Epidemic Diarrhea Virus (PEDV) S1 Region Combined With Lactobacillus-expressed N Protein. Appl Microbiol Biotechnol. 2012;93(6):2437-46.

33. Oh J, Lee KW, Choi HW, Lee C. Immunogenicity and Protective Efficacy of Recombinant S1 Domain of the Porcine Epidemic Diarrhea Virus Spike Protein. Arch Virol. 2014;159(11):2977-87.

34. Wang $C$, Yan F, Zheng $X$, Wang $H$, Jin H, Wang C, Zhao Y, Feng N, Wang T, Gao Y, Yang S, Xia X. Porcine Epidemic Diarrhea Virus Virus-Like Particles Produced in Insect Cells Induce Specific Immune Responses in Mice. Virus Genes. 2017;53(4):548-54.

35. Kost TA, Kemp CW. Fundamentals of Baculovirus Expression and Applications. Adv Exp Med Biol. 2016;896:187-97.

36. Chen P, Wang K, Hou Y, Li H, Li X, Yu L, Jiang Y, Gao F, Tong W, Yu H, Yang Z, Tong G, Zhou Y. Genetic Evolution Analysis and Pathogenicity Assessment of Porcine Epidemic Diarrhea Virus Strains Circulating in Part of China During 2011-2017. Infect Genet Evol. 2019;69:153-65.

37. Tiwari P, Saini S, Upmanyu S, Benjamin B, Tandon R, Saini KS, Sahdev S. Enhanced Expression of Recombinant Proteins Utilizing a Modified Baculovirus Expression Vector. Mol Biotechnol. 2010;46(1):80-9.

38. Khan MA, Hassan N, Ahmad N, Khan MI, Zafar AU, Khan F, Husnain T. Studies to Analyse the Relationship Between IFNa2b Gene Dosage and Its Expression, Using a Pichia Pastoris-Based Expression System. Yeast. 2014;31(1):13-28.

39. Hohenblum H, Gasser B, Maurer M, Borth N, Mattanovich D. Effects of Gene Dosage, Promoters, and Substrates on Unfolded Protein Stress of Recombinant Pichia Pastoris. Biotechnol Bioeng. 2004;85(4):367-75.

40. Okda FA, Lawson S, Singrey A, Nelson J, Hain KS, Joshi LR, Christopher-Hennings J, Nelson EA, Diel DG. The S2 Glycoprotein Subunit of Porcine Epidemic Diarrhea Virus Contains Immunodominant Neutralizing Epitopes. Virology. 2017;509:185-94.

\section{Figures}




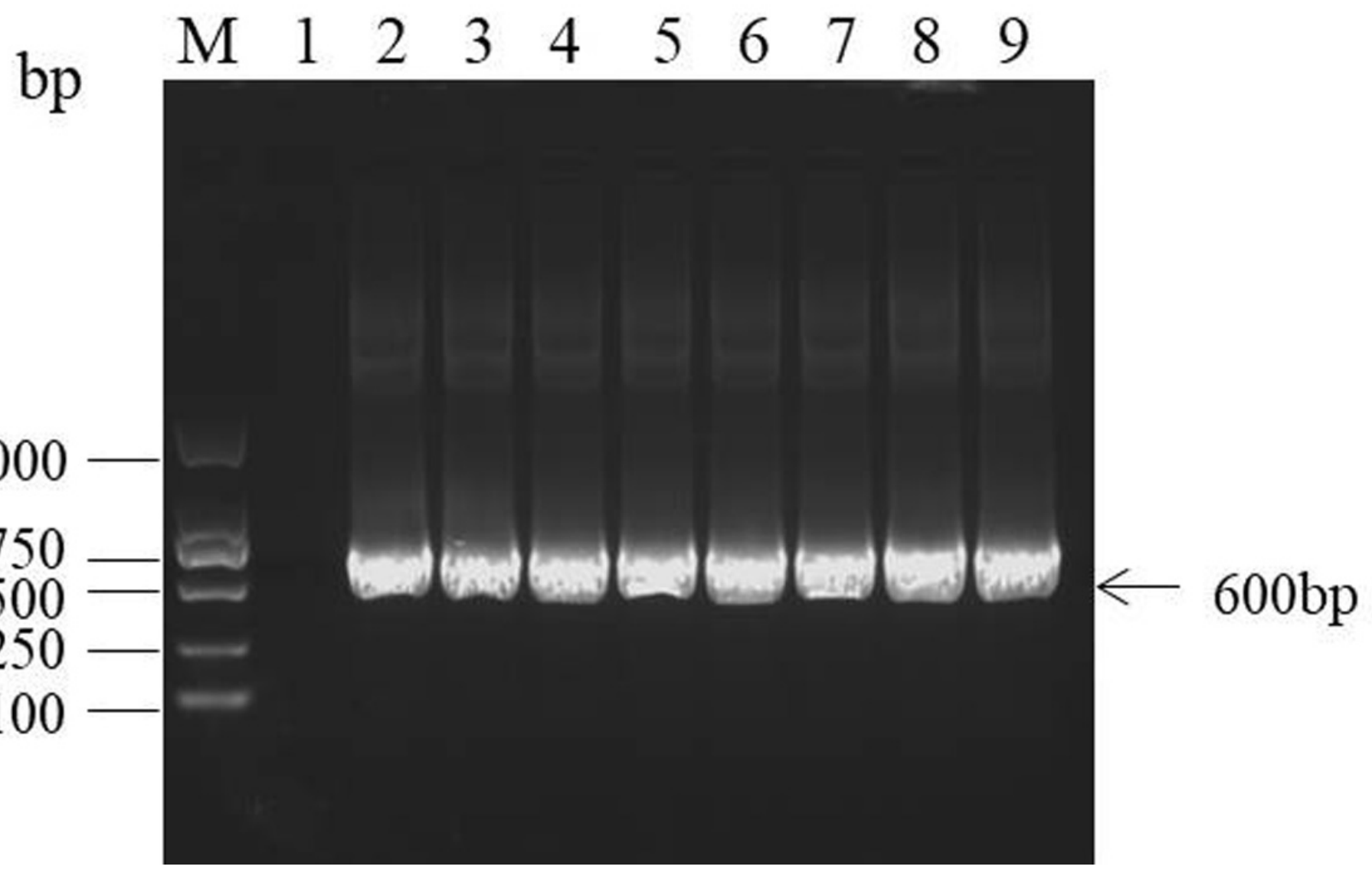

\section{Figure 1}

Identification of the baculoviral genomic DNA samples by PCR. Lane M: DL2000 Marker; Lane 1: negative control, genomic DNA of uninfected Sf9 cells; Lane 2 Lane 9: genomic DNA of Sf9 cells infected with the recombinant virus Ac-IM-p10-S1CD, Ac-IM-2p10-2S1CD, Ac-IM-3p10-3S1CD, Ac-IM-ph-S1CD, Ac-IM2ph-2S1CD, Ac-IM-3ph-3S1CD, Ac-IM-php10-2S1CD, and Ac-IM-2ph2p10-4S1CD, respectively.

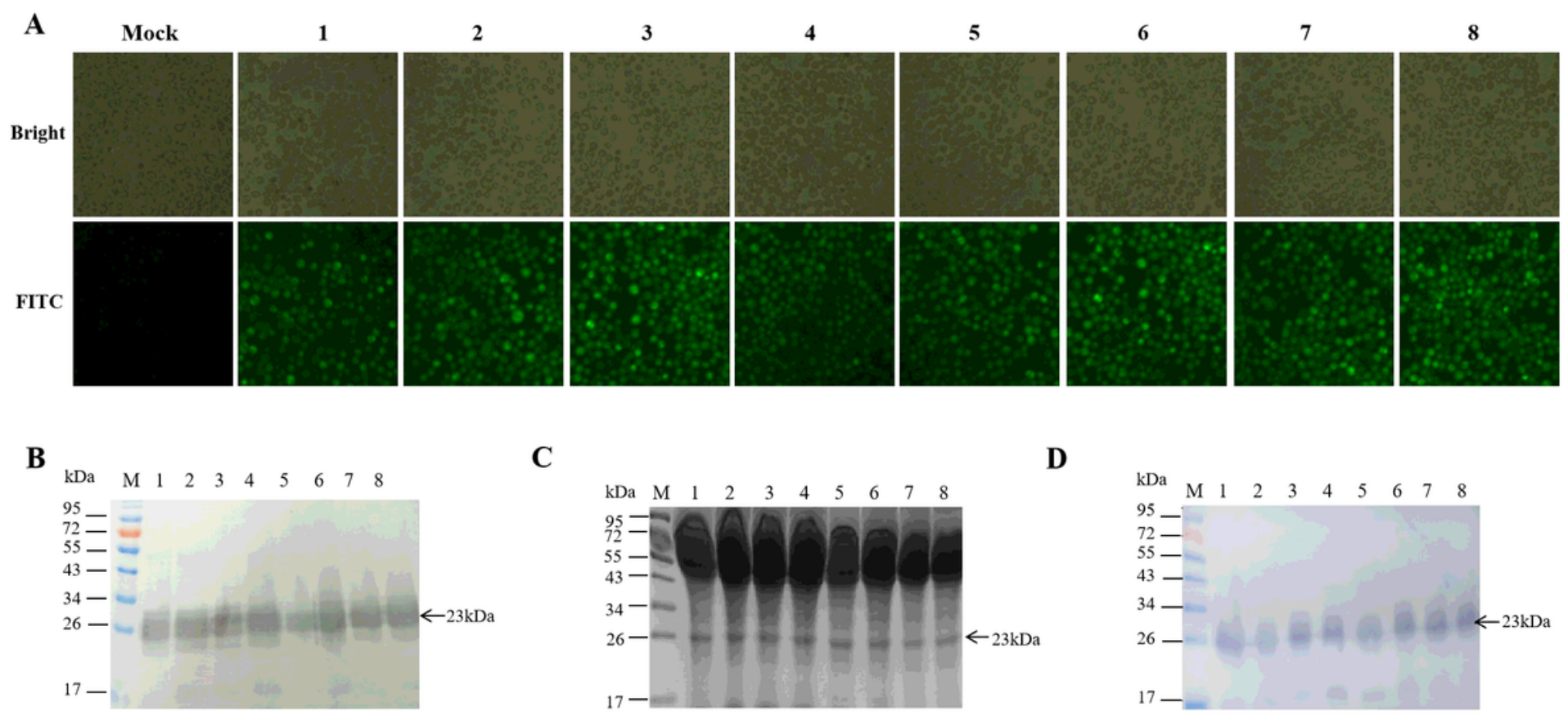


Figure 2

. Analysis of the expression of S1CD protein using baculovirus expression system. (A) IFA analysis of S1CD expression in Sf9 cells infected with different baculoviruses at $48 \mathrm{~h}$ p.i. (200x) (B) Western blot analysis of S1CD expression in Sf9 cells infected with different baculoviruses at $96 \mathrm{~h}$ p.i. (C) SDS-PAGE analysis of S1CD expression in culture supernatant of Sf9 cells infected with different baculoviruses at $96 \mathrm{~h}$ p.i. (D) Western blot analysis of S1CD expression in culture supernatant of Sf9 cells infected with different baculoviruses at 96 h p.i. Mock: Samples from the un-infected Sf9 cells; $1 \sim$ 8: Samples from the Sf9 cells infected with the recombinant virus Ac-IM-p10-S1CD, Ac-IM-2p10-2S1CD, Ac-IM-3p10-3S1CD, Ac-IM-ph-S1CD, Ac-IM- 2ph-2S1CD, Ac-IM-3ph-3S1CD, Ac-IM-php10-2S1CD, and Ac-IM-2ph2p10-4S1CD, respectively.

A

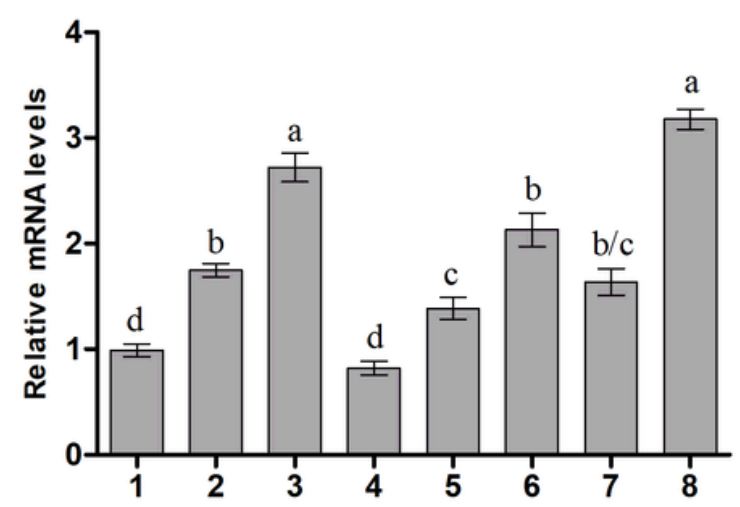

B

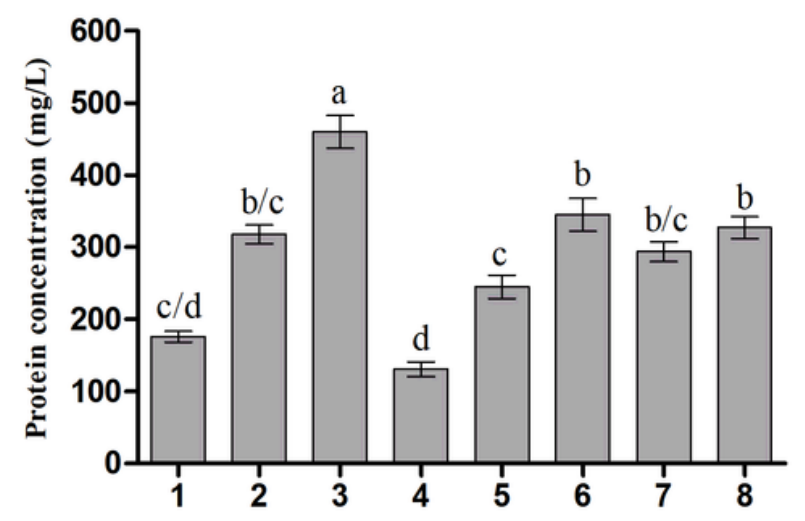

\section{Figure 3}

Analysis of the expression level of S1CD gene in Sf9 cells. (A) Analysisof the transcriptional level (mRNA) by qPCR. (B) Analysis of the protein expression level by ELISA detection kit. Data are shown as the mean \pm standard error value from three independent experiments. One-way ANOVA followed by Tukey's post hoc test was used to determine significant difference between groups. When two sets of data are labeled with superscripts of different letters, it indicates that these sets of data are statistically different $(\mathrm{P}<$ 0.05). $1 \sim 8$ : Samples from the Sf9 cells infected with the recombinant baculovirus Ac-IM-p10-S1CD, AcIM-2p10-2S1CD, Ac-IM-3p10-3S1CD, Ac-IM-ph-S1CD, Ac-IM-2ph-2S1CD, Ac-IM-3ph-3S1CD, Ac-IM-php102S1CD, and Ac-IM-2ph2p10-4S1CD, respectively. 
$\mathrm{kDa}$

$\mathrm{M}$

$1 \quad 2$

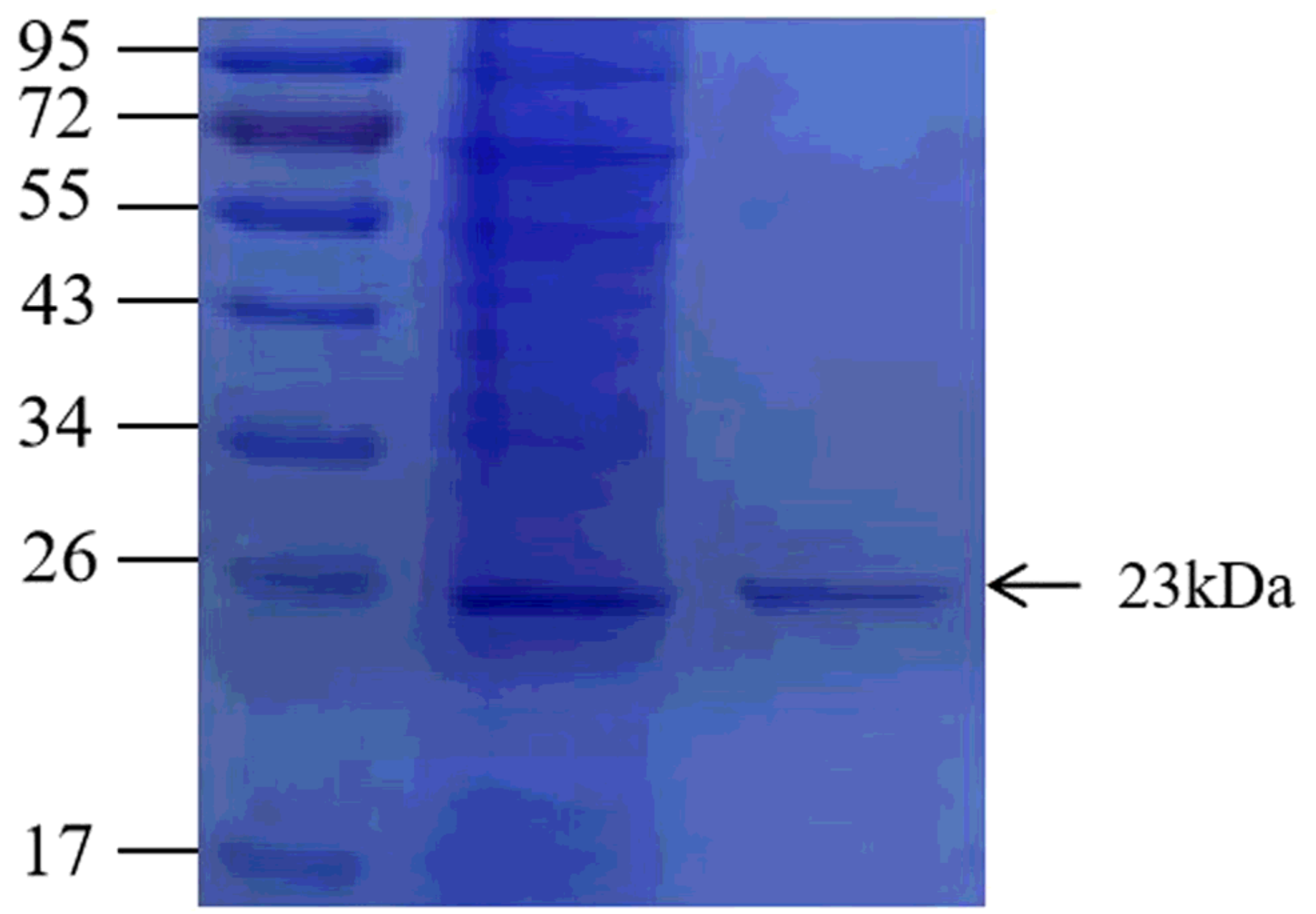

Figure 4

SDS-PAGE analysis of purified recombinant S1CD protein. Lane M: Pageruler ${ }^{\mathrm{TM}}$ Prestained Protein Ladder, 10 to $180 \mathrm{kDa}$; Lane 1: ultrasound supernatant of the recombinant baculovirus Ac-IM-3p10-3S1CD infected Sf9 cells; Lane 2: 300 mM Imidazole eluent.

A

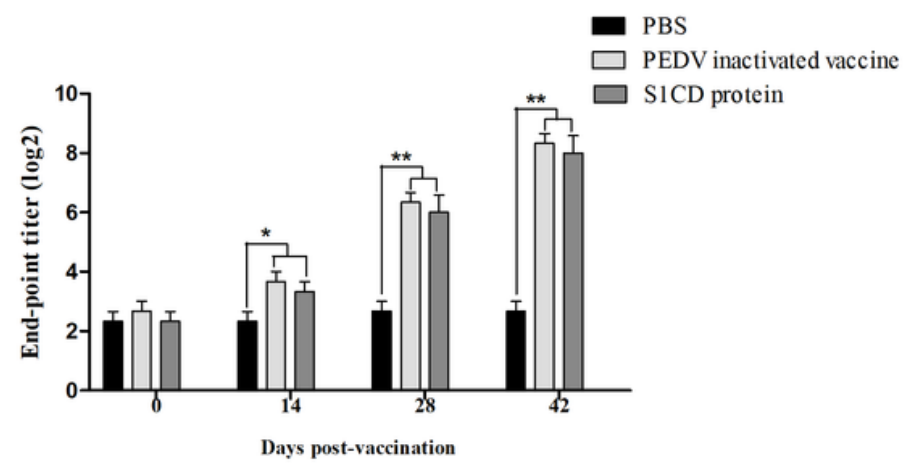

B

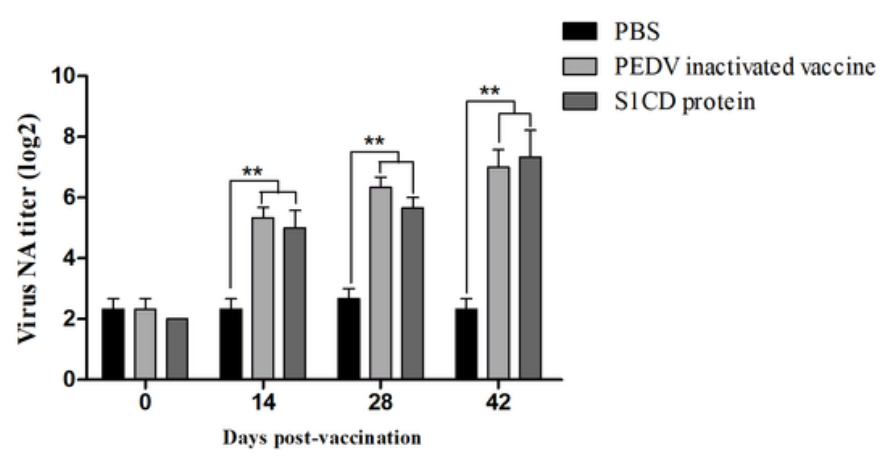




\section{Figure 5}

Immunogenicity of purified S1CD protein in mice. BALB/c mice were vaccinated with purified S1CD protein, commercial PEDV inactivated vaccine or PBS three times on days 0,14 , and 28. (A) Anti-PEDV specific IgG antibody levels in the sera. End-point titer (log2) were defined as the highest dilution yielding an absorbance that was two times higher than the background for the serum samples. (B) Virus NA levels in the sera. Results represent the mean \pm standard error value of triplicates. One-way ANOVA followed by Tukey's post hoc test was used to determine significant difference between groups ( ${ }^{\star} p<0.05$ and ${ }^{* \star} p<$ $0.01)$.

A

COE(140aa) Linker D56(28aa) Linker 6His

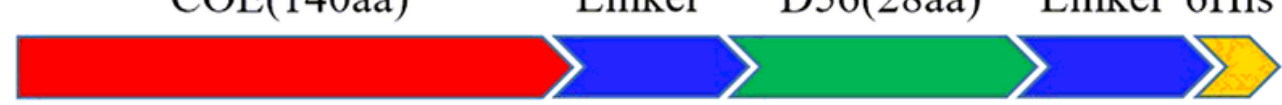

B

MSGGTLPSFNDHSFVNITVSAAFGGHSGANLIASDTTINGFSSFC VDTRQFTITLFYNVTNSYGYVSKSQDSNCPFTLQSVNDYLSFSKF CVSTSLLAGACTIDLFGYPEFGSGVKFTSLYFQFTKGELITGTPKP LQGVTDVSGGGGSGGGGSGGGGSVLVYSNIGVCKSGSIGYVPLQ DGQVKIAGGGGSGGGGSGGGGSHHHHHH.

Figure 6

Design of S1CD Fusion protein. (A) Diagram of S1CD containing COE gene of PEDV, linker (GGGGS)3, S1D 5 and S1D 6 gene of PEDV, and $6 \times$ His tag. S1CD has 205 amino acids with a PI of 6.90 and MW of $23.0 \mathrm{kDa}$. (B) Amino acid sequence of $\mathrm{S} 1 \mathrm{CD}$; sequence of $\mathrm{COE}$ is marked in red; sequence of the linker is marked in blue; sequence of the S1D56 protein is marked in green; and sequence of $6 \times$ His tag is marked in orange. 


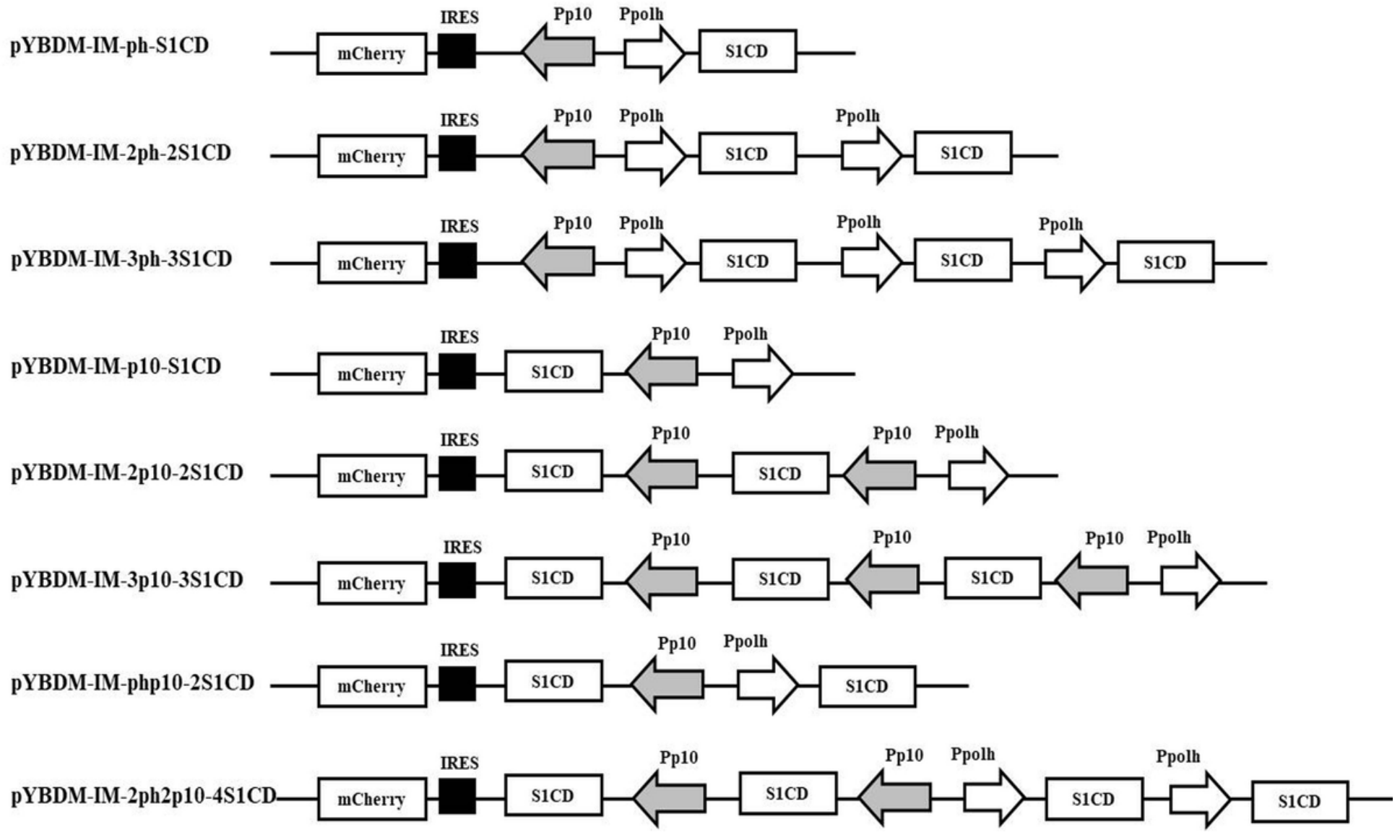

Figure 7

Schematic diagram of different transfer vectors containing PEDV S1CD gene. polh: polyhedrin promoter; p10: p10 promoter; IRES: internal ribosome entry site derived from Rhopalosiphum padi virus; mCherry: cDNA of red fluorescent protein derived from mushroom coral.

\section{Supplementary Files}

This is a list of supplementary files associated with this preprint. Click to download.

- Additionalfile1.docx

- ChecklistS1.docx 